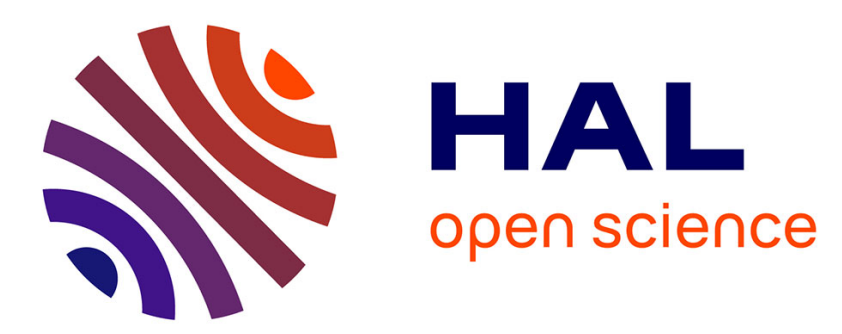

\title{
Thermal quadrupole method with internal heat sources
}

Jérôme Pailhes, Christophe Pradere, Jean-Luc Battaglia, Jean Toutain, Andrzej Kusiak, Waste Aregba, Jean-Christophe Batsale

\section{To cite this version:}

Jérôme Pailhes, Christophe Pradere, Jean-Luc Battaglia, Jean Toutain, Andrzej Kusiak, et al.. Thermal quadrupole method with internal heat sources. International Journal of Thermal Sciences, 2012, 53, pp.49-55. 10.1016/j.ijthermalsci.2011.10.005 . hal-01020781

\section{HAL Id: hal-01020781 \\ https://hal.science/hal-01020781}

Submitted on 8 Jul 2014

HAL is a multi-disciplinary open access archive for the deposit and dissemination of scientific research documents, whether they are published or not. The documents may come from teaching and research institutions in France or abroad, or from public or private research centers.
L'archive ouverte pluridisciplinaire HAL, est destinée au dépôt et à la diffusion de documents scientifiques de niveau recherche, publiés ou non, émanant des établissements d'enseignement et de recherche français ou étrangers, des laboratoires publics ou privés. 


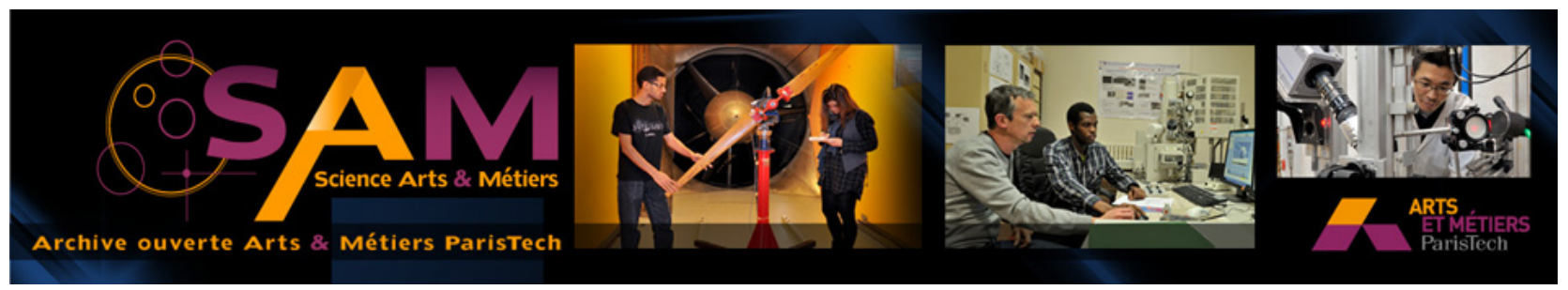

\section{Science Arts \& Métiers (SAM)}

is an open access repository that collects the work of Arts et Métiers ParisTech researchers and makes it freely available over the web where possible.

This is an author-deposited version published in: http://sam.ensam.eu

Handle ID: .http://hdl.handle.net/10985/8331

\section{To cite this version :}

Jérôme PAILHES, Christophe PRADERE, Jean-Luc BATTAGLIA, Jean TOUTAIN, Andrzej KUSIAK, Waste AREGBA, Jean-Christophe BATSALE - Thermal quadrupole method with internal heat sources - International Journal of Thermal Sciences - Vol. 53, p.49-55 - 2012 


\title{
Thermal quadrupole method with internal heat sources
}

\author{
J. Pailhes*, C. Pradere, J.-L. Battaglia, J. Toutain, A. Kusiak, A.W. Aregba, J.-C. Batsale \\ TREFLE, UMR CNRS 8508, Université Bordeaux 1, Arts et Métiers ParisTech, Esplanade des Arts et Métiers, 33405 Talence, France
}

Keywords:

Thermal quadrupoles

Numerical simulation

Heat source

Multilayer materials

\begin{abstract}
A B S T R A C T
A new method based on the thermal quadrupoles technique for heat transfer modelling in multilayered slabs with heat sources is proposed. Classical thermal quadrupoles use hyperbolic functions and numerical problems occur according to the argument value that depends on thermophysical and geometrical properties as well as characteristics times. We propose a new formulation based on exponential function with negative argument. Using this formulation in the classical equivalent impedance network allows to compute efficiently the thermal behaviour of multilayered slabs with internal heat sources whatever the time and the thermophysical properties. This approach is applied in order to simulate heat transfer in three different multilayered materials with heat sources. These simulations show the capability of such a methodology to simulate time and space multiscale heat diffusion problems.
\end{abstract}

\section{Introduction}

The quadrupole method is a well known analytical tool in heat transfer modelling. It is an exact method to predict temperature in time-varying linear systems [1]. This method allows to assemble multilayered slabs with different geometrical and thermophysical properties. The integral transforms are at the basis of the method. Laplace or Fourier transforms are used depending on the transient regime. However, it is always possible to switch from one to the other by only replacing the Laplace variable with $j \omega$, where $\omega$ if the frequency and $j=\sqrt{-1}$ is the complex argument. Additional integral transforms, as the Fourier or Hankel ones, can also be efficiently used in case of multidimensional heat transfer [2]. The only constraint relates to symmetry considerations. The quadrupole method has found several applications in the field of thermal properties measurement in one-dimensional transfer [3-6] as well as in multidimensional one [7-9]. A lot of studies use the quadrupole formalism in order to estimate the thermal diffusivity and effusivity of single layer [10-13] or multilayered [14]. It has been used also to obtain simplified solution for heat transfer in material forming processes [15]. This technique was used to estimate phononic thermal properties in semi transparent medium with heat source [16]. Several applications where heat source occurred inside the medium have been presented $[17,18]$.

Even if this technique received a lot of attention in the past, some practical aspects remained unsolved. This paper aims to

\footnotetext{
* Corresponding author. Tel./fax: +33 556844422 .

E-mail address: jerome.pailhes@ensam.eu (J. Pailhes).
}

focus on two specific cases. The first one concerns practical computation of the quadrupole components when the argument in the hyperbolic function becomes too high. As a consequence, the numerical computation of the function is impossible. This configuration comes to investigate the solution of heat transfer at small times in a thick material (without making the assumption of the semi-infinite behaviour). The second case concerns the processing of a heat source term in the material and the way to take it into account in the quadrupole formalism. The solution presented in the literature [1], although it is exact, presents numerical drawbacks especially in case of a multilayered media.

For both cases, it is presented a solution that allows keeping the meaningful simplicity of the quadrupole method. An application on multilayer materials is presented. Thickness and thermal properties as well as heat source location lead to difficult numerical implementation in case of the use of the classical quadrupole method. It is shown that the new formulation presented in this paper allows to overcome these difficulties.

\section{General solution for multilayered samples with internal heat sources}

Let us consider one-dimensional heat transfer in a wall, of thickness $e$, thermal conductivity $\lambda$ and thermal diffusivity $a$. It is also considered an internal heat source function $g(z, t)$ as well as a prescribed heat flux density and heat exchange, characterized by the thermal exchange coefficient $h$, at both sides of the wall. Thereby, heat transfer is mathematically described as: 


\section{Nomenclature}

$T \quad$ Temperature (K)

a Thermal diffusivity $\left(\mathrm{m}^{2} \mathrm{~s}^{-1}\right)$

$\rho \quad$ Density $\left(\mathrm{kg} \mathrm{m}^{-3}\right)$

$c \quad$ Heat capacity (W s kg ${ }^{-1} \mathrm{~K}^{-1}$ )

$\lambda$ Thermal conductivity $\left(\mathrm{W} \mathrm{m}^{-1} \mathrm{~K}^{-1}\right)$

$g \quad$ Heat source $\left(\mathrm{W} \mathrm{m}^{-3}\right)$

$G \quad$ Laplace transform of the heat source $\left(\mathrm{W} \mathrm{m}^{-3}\right)$

$t \quad$ Time $(\mathrm{s})$

$z \quad$ Space location (1D) (m)

$\varphi_{0}(t) \quad$ Front heat flux density $\left(\mathrm{W} \mathrm{m}^{-2}\right)$

$\varphi_{e}(t) \quad$ Rear heat flux density $\left(\mathrm{W} \mathrm{m}^{-2}\right)$

$\psi_{0}(p) \quad$ Laplace transform of Front Heat Flux density $\left(\mathrm{W} \mathrm{m}^{-2}\right)$

$\psi_{e}(p) \quad$ Laplace transform of Rear Heat Flux density $\left(\mathrm{W} \mathrm{m}^{-2}\right)$

$H_{F}$
$H_{R}$

e

$\theta$

$p$

$K_{1}, K_{2}$

$y(z, p)$

$\Phi$

A

$B$

C

$D$

$X$

$Y$

$Z_{1}, Z_{2}$

$Z_{3}$

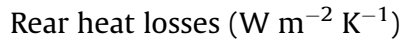

Thickness (m)

Laplace transform of the temperature (K)

Laplace variable $\left(\mathrm{s}^{-1}\right)$

Constants (No dimension)

Particular solution (K)

Laplace transform of the heat flux density $\left(\mathrm{W} \mathrm{m}^{-2}\right)$

Quadrupole Coefficient (No dimension)

Quadrupole Coefficient $\left(\mathrm{K} \mathrm{m}^{2} \mathrm{~W}^{-1}\right)$

Quadrupole Coefficient ( $\mathrm{W} \mathrm{m} \mathrm{m}^{-2} \mathrm{~K}^{-1}$ )

Quadrupole Coefficient (No dimension)

Voltage generator for the quadrupole (K)

Current source for the quadrupole $\left(\mathrm{W} \mathrm{m}^{-2}\right)$

Thermal Impedance (No dimension)

Thermal Impedance $\left(\mathrm{K} \mathrm{m}^{2} \mathrm{~W}^{-1}\right)$

$$
\left\{\begin{array}{l}
\frac{\partial^{2} T(z, t)}{\partial z^{2}}+\frac{1}{\lambda} g(z, t)=\frac{1}{a} \frac{\partial T(z, t)}{\partial t}, \text { for } 0<z<e \text { and } t>0 \\
T(z, t=0)=0 \\
-\left.\lambda \frac{\partial T(z, t)}{\partial z}\right|_{z=0}=\varphi_{0}(t)-h_{F} T(z=0, t) \\
-\left.\lambda \frac{\partial T(z, t)}{\partial z}\right|_{z=e}=\varphi_{e}(t)+h_{R} T(z=e, t)
\end{array}\right.
$$

As it is reported in [1], the Laplace integral transform is applied on the time variable for both temperature and heat flux. In case of the temperature as the considered quantity, it is written as:

$\theta(z, p)=L[T(z, t)]=\int_{t=0}^{+\infty} T(z, t) \exp (-p t) d t$

Considering $G$ and $\psi$ as the Laplace transform of $g$ and $\varphi$ respectively and applying the Laplace transform upon the set of equations in (1), it is obtained:

$\left\{\begin{array}{l}\frac{d^{2} \theta(z, p)}{d z^{2}}+\frac{1}{\lambda} G(z, p)=\frac{p}{a} \theta(z, p) \text { for } 0<z<e \quad(a) \\ -\left.\lambda \frac{d \theta(z, p)}{d z}\right|_{z=0}=\Psi_{0}(p)-h_{F} \theta(z=0, p)=\Phi_{0}(p) \\ -\left.\lambda \frac{d \theta(z, p)}{d z}\right|_{z=e}=\Psi_{e}(p)+h_{R} \theta(z=e, p)=\Phi_{e}(p)\end{array}\right.$

The general solution of (3). (a) is of the following form:

$\theta(z, p)=K_{1} \cosh (\beta z)+K_{2} \sinh (\beta z)+y(z, p)$, with $\beta=\sqrt{\frac{p}{a}}$

Constants $K_{1}$ and $K_{2}$ are determined from boundary conditions (3).(b) and (3).(c) and the particular solution $y(z, p)$ is deduced from the expression of the source term $G$. Using the quadrupole formalism developed in [1] from relation (4), the transformed heat flux $\Phi_{0}=\Phi(z=0, p)$ and temperature $\theta_{0}=\theta(z=0, p)$ can be expressed according to the quantities $\Phi_{e}=\Phi(z=e, p)$ and $\theta_{e}=\theta(z=e, p)$, as:

$\left\{\begin{array}{c}\theta_{0} \\ \Phi_{0}\end{array}\right\}=\left[\begin{array}{ll}A & B \\ C & D\end{array}\right]\left\{\begin{array}{c}\theta_{e} \\ \Phi_{e}\end{array}\right\}-\left[\begin{array}{c}X \\ Y\end{array}\right]$ with:

$A=D=\cosh (\beta e), B=\frac{\sinh (\beta e)}{\lambda \beta}, C=\lambda \beta \sinh (\beta e)$,

and:

$X=\int_{0}^{e}\left(\frac{1}{\lambda} G(z, p)\right) \frac{\sinh (\beta z)}{\beta} d z, Y=\int_{0}^{e} G(z, p) \cosh (\beta z) d z$

$A, B, C$ and $D$ are the components of the thermal quadrupole for a passive wall whereas the internal source is involved in $X$ and $Y$ terms. It is largely used with the classical equivalent electrical network represented in Fig. 1, where $X$ and $Y$ are respectively a voltage generator and a current generator. The analytical expressions of the thermal impedances are:

$Z_{1}=\frac{A-1}{C}, Z_{2}=\frac{D-1}{C}, Z_{3}=\frac{1}{C}$

For practical reasons, we will consider only the case where $G(z$, $p$ ) is not depending on $z$. Nevertheless, it is possible to approximate a $z$-continuous function $G(z, p)$ by a discrete layered distribution. $G(z, p)$ can be considered constant for each layer $i$ and with this assumption we obtain :

$\left.X^{i}=\frac{A^{i}-1}{C^{i}}\right) Y^{i}$

An additional Laplace inverse transform is also required to retrieve the temperature field in the real space.

One of the most interesting features of the quadrupoles method is its direct application to multilayered materials as a composite material. The solution for multilayered materials is detailed in $[1,19]$.

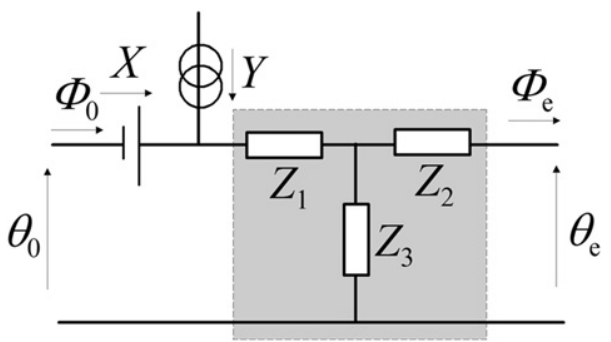

Fig. 1. Wall with internal source [1]. 
A generalization of this solution is obtained in $3 \mathrm{D}$ and $2 \mathrm{D}$ axisymmetric heat transfer geometrical configurations using the Fourier and Hankel transforms respectively (see reference [2,19]). In such configurations, relations (5)-(7) remain unchanged but $\beta$ is modified $[1,2,20]$.

As it is viewed in relation (6) the quadrupoles terms are defined from hyperbolic functions. However, numerical problems occur using his formulation according to the argument value, namely $\beta e$. In order to avoid such problems we propose to use an original formulation of these terms.

\section{The use of the exponential formulation in quadrupole formalism}

\subsection{Drawback with hyperbolic functions}

Numerical computation of the quadrupole terms from relations (6) and (7) rests on the use of hyperbolic functions that are calculated using their general expressions with the exponential

$$
\begin{aligned}
\left\{\begin{array}{c}
\theta_{0} \\
\Psi_{0}(p)-h_{F} \theta_{0}
\end{array}\right\} & =\left[\begin{array}{l}
\exp (\beta e) \\
\exp (\beta e)
\end{array}\right]\left(\left[\begin{array}{cc}
\tilde{A} & \tilde{B} \\
\tilde{C} & \tilde{D}
\end{array}\right]\left\{\begin{array}{c}
\theta_{e} \\
\Psi_{e}(p)+h_{R} \theta_{e}
\end{array}\right\}\right. \\
& \left.-\left[\begin{array}{c}
\frac{\tilde{A}-\exp (-\beta e)}{\tilde{C}} Y \exp (-\beta e) \\
Y \exp (-\beta e)
\end{array}\right]\right)
\end{aligned}
$$

With:

$$
\begin{aligned}
& \tilde{A}=\frac{1+\exp (-2 \beta e)}{2}=\tilde{D} \\
& \tilde{B}=\frac{1-\exp (-2 \beta e)}{2 \lambda \beta} \\
& \tilde{C}=\lambda \beta \frac{1-\exp (-2 \beta e)}{2}
\end{aligned}
$$

The numerical computation of $(\tilde{A}, \tilde{B}, \tilde{C}, \tilde{D})$ is suitable whatever the value of $a, t$ and $e$. As an example, this allows the calculation of the temperature at the front face $(z=0)$ as:

$\theta_{0}=\frac{\left.\left(\tilde{A}+h_{R} \tilde{B}\right) \Psi_{0}(p)-\Psi_{e}(p) \exp (-\beta e)+Y \exp (-\beta e) \quad 1+h_{R} \frac{\tilde{A}-\exp (-\beta e)}{\tilde{C}}\right)}{h_{F}\left(\tilde{A}+h_{R} \tilde{B}\right)+\tilde{C}+h_{R} \tilde{A}}$

Whereas, the solution at the rear face $(z=e)$ is given by:

$\theta_{e}=\frac{\left.-\left(\tilde{A}+h_{F} \tilde{B}\right) \Psi_{e}(p)+\Psi_{0}(p) \exp (-\beta e)+Y \exp (-\beta e) 1+h_{F} \frac{\tilde{A}-\exp (-\beta e)}{\tilde{C}}\right)}{h_{F}\left(\tilde{A}+H_{R} \tilde{B}\right)+\tilde{C}+h_{R} \tilde{A}}$

function $(\cosh (x)=(\exp (x)+\exp (-x)) / 2, \sinh (x)=(\exp (x)-$ $\exp (-x)) / 2$ ). The validity domain for the argument in double precision must not be greater than 700 . It means that one must verify $e \sqrt{p / a}<700$ for each considered value of the Laplace variable $p$. As an example, let us consider material with $a=10^{-7} \mathrm{~m}^{2} \mathrm{~s}^{-1}$ and $t=1 \mathrm{~s}$. It appears that the thickness must not be greater that some millimetres in order to compute $\exp [e \sqrt{p / a}]$. These computations appear when problems are not well posed in the sense of Hadamard [21].

\subsection{Use of another exponential formulation - application to the wall}

It has been viewed in the previous section the general solution for the one-dimensional heat transfer diffusion problem in a wall with heat source on the form of the relation (5). Drawback on the exponential computation when the argument is more than 700 can be overcome by rewriting the hyperbolic functions as follow:

$$
\begin{aligned}
& \cosh (\beta e)=\exp (\beta e)\left(\frac{1+\exp (-2 \beta e)}{2}\right) \\
& \sinh (\beta e)=\exp (\beta e)\left(\frac{1-\exp (-2 \beta e)}{2}\right)
\end{aligned}
$$

By substituting these expressions in components $A, B$ and $C$ of the quadrupole in the relation (5), it is obtained:
There is no exponential function with positive argument $\beta_{e}$ in relation (13) and (14). Although the computation of $\theta_{e}$ and $\theta_{0}$ from relations (13) or (14) does not involve any problems considering a uniform heat source $G$, the generalization for multilayered configurations starting from relation (11) will not lead to a comparable analytical formalism. Therefore, A new impedance network representation for heat sources is proposed.

\section{Use of exponential and impedance formulation in quadrupole problem with heat sources}

\subsection{Impedance formulation for heat sources}

An equivalent electrical network to that represented in Fig. 1 is presented in Fig. 2. In this new representation the voltage generator disappears and the heat source $G$ is represented as a current source $Y^{\prime}$ located at the node of the cell constituted from the three impedances $Z_{1}^{\prime}, Z_{2}^{\prime}$ et $Z_{3}^{\prime}$. Since $G$ is uniform, this symmetrical representation seems natural but we demonstrate right now that it is fully exact. Indeed, one has:

$\Phi_{0}+Y^{\prime}=\frac{1}{Z_{3}^{\prime}} \theta^{i}+\Phi_{e}$

Furthermore:

$\Phi_{0}=\frac{1}{Z_{1}^{\prime}}\left(\theta_{0}-\theta^{i}\right)$, 


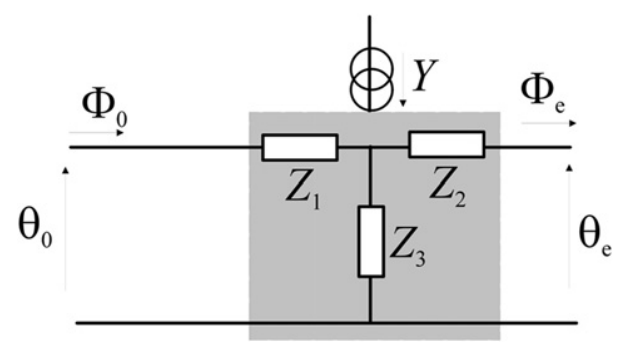

Fig. 2. Wall with internal source with the new formalism.

and:

$\Phi_{e}=\frac{1}{Z_{2}^{\prime}}\left(\theta^{i}-\theta_{e}\right)$

By using relations (15)-(17) it is finally obtained:

$$
\left\{\begin{array}{c}
\theta_{0}=\frac{Z_{3}^{\prime}+Z_{1}^{\prime}}{Z_{3}^{\prime}} \theta_{e}+\left(\frac{Z_{2}^{\prime} Z_{3}^{\prime}+Z_{1}^{\prime} Z_{2}^{\prime}+Z_{1}^{\prime} Z_{3}^{\prime}}{Z_{3}^{\prime}}\right) \Phi_{e}-Z_{1}^{\prime} Y^{\prime} \\
\Phi_{0}=\frac{1}{Z_{3}^{\prime}} \theta_{e}+\left(\frac{Z_{2}^{\prime}+Z_{3}^{\prime}}{Z_{3}^{\prime}}\right) \Phi_{e}-Y^{\prime}
\end{array}\right.
$$

By comparing relation (18) with relation (5), we are able to express the quadrupole terms as:

$$
\begin{aligned}
& A=\frac{Z_{3}^{\prime}+Z_{1}^{\prime}}{Z_{3}^{\prime}}, B=\frac{Z_{2}^{\prime} Z_{3}^{\prime}+Z_{1}^{\prime} Z_{2}^{\prime}+Z_{1}^{\prime} Z_{3}^{\prime}}{Z_{3}^{\prime}}, C=\frac{1}{Z_{3}^{\prime}}, D=\frac{Z_{2}^{\prime}+Z_{3}^{\prime}}{Z_{3}^{\prime}} \\
& Y^{\prime}=Y, X=Z_{1}^{\prime} Y^{\prime}
\end{aligned}
$$

Since the determinant of the quadrupole is equal to 1 $(A D-B C=1)$, it is thus possible to express $Z_{1}^{\prime}, Z_{2}^{\prime}$ et $Z_{3}^{\prime}$ and current source $Y^{\prime}$ as:

$Z_{1}^{\prime}=Z_{2}^{\prime}=\frac{A-1}{C}=Z_{1}=Z_{2}, Z_{3}^{\prime}=\frac{1}{C}=Z_{3}, Y^{\prime}=\frac{X}{Z_{1}^{\prime}}$

Using the quadrupole formalism the relation (5) becomes with the new network:

$\left\{\begin{array}{l}\theta_{0} \\ \Phi_{0}\end{array}\right\}=\left[\begin{array}{ll}A & B \\ C & D\end{array}\right]\left\{\begin{array}{l}\theta_{e} \\ \Phi_{e}\end{array}\right\}-\left[\left(\frac{A-1}{C}\right) Y\right]$

As argued before, it is thus found that thermal impedances values remain exactly the same for the two electrical networks represented in Figs. 1 and 2 respectively. For the current source term $Y^{\prime}=Y$ if $G$ does not vary according to $z$, this condition is always satisfied. However, this condition is not absolutely restrictive. Indeed, in case of a $z$-dependent heat source, it is always possible to slip the layer as many times as required in order to reconstruct the heat source term in a discrete way (heat source in each layers being uniform).

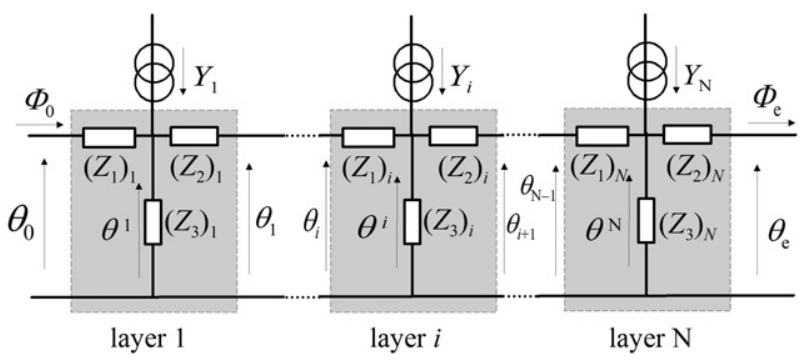

Fig. 3. Multilayer with internal sources.

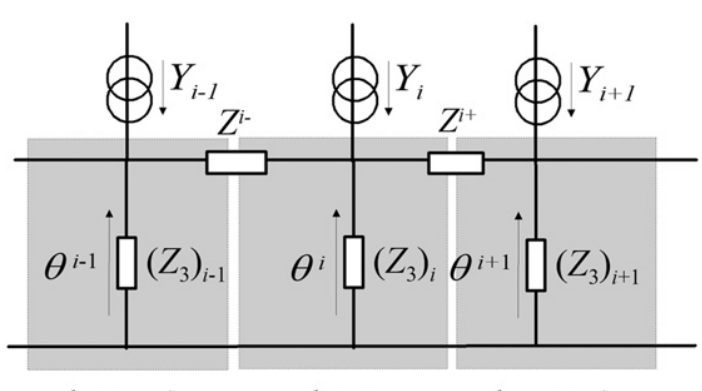

layer $i-1 \quad$ layer $i \quad$ layer $i+1$

Fig. 4. Wall with multilayer and internal sources (simplified formalism).

\subsection{Application to multilayered systems}

Let us consider a composite material constituted from $N$ successive layers whose thermal properties are known. A heat source is defined in each layer. It is admitted here that the contact is perfect. However, a thermal boundary resistance could have been taken into account within this formalism on the form of a pure resistance between each cell. From Fig. 3, it can be expressed the current conservation law at the central node for layer $i$ as:

$\frac{1}{\left(Z_{1}\right)_{i}}\left(\theta_{i}-\theta^{i}\right)+Y_{i}=\frac{1}{\left(Z_{3}\right)_{i}} \theta^{i}+\frac{1}{\left(Z_{2}\right)_{i}}\left(\theta^{i}-\theta_{i+1}\right)$

It must be emphasized (see Fig. 4) that $\theta^{i}$ has no physical meaning: it is not the temperature $\theta_{i}$ at interface $i$ nor the average temperature of layer $i$. To reduce the number of equations, it is only applied on temperatures at central nodes (Fig. 4). Thereby, relation (22) becomes:

$\frac{1}{Z^{i-}}\left(\theta^{i-1}-\theta^{i}\right)+Y_{i}=\frac{1}{\left(Z_{3}\right)_{i}} \theta^{i}+\frac{1}{Z^{i+}}\left(\theta^{i}-\theta^{i+1}\right)$

With:

$Z^{i-}=\left(Z_{2}\right)_{i-1}+\left(Z_{1}\right)_{i}$ and $Z^{\mathrm{i}+}=\left(Z_{2}\right)_{i}+\left(Z_{1}\right)_{i+1}$

Considering the discussion about the hyperbolic functions calculation according to the exponential function argument in section 2, relation (23) is written on the following equivalent form:

$$
\begin{gathered}
\frac{\exp \left(-\beta_{i} e_{i}\right)}{Z^{i-}}\left(\theta^{i-1}-\theta^{i}\right)+Y_{i} \exp \left(-\beta_{i} e_{i}\right) \\
=\frac{1}{\left(\tilde{Z}_{3}\right)_{i}} \theta^{i}+\frac{\exp \left(-\beta_{i} e_{i}\right)}{Z^{i+}}\left(\theta^{i}-\theta^{i+1}\right)
\end{gathered}
$$

With:

$$
\begin{aligned}
& \tilde{Z}^{i-}=Z^{i-}=\frac{\tilde{D}_{i-1}-\exp \left(-\beta_{i-1} e_{i-1}\right)}{\tilde{C}_{i-1}}+\frac{\tilde{A}_{i}-\exp \left(-\beta_{i} e_{i}\right)}{\tilde{C}_{i}} \\
& \tilde{Z}^{i+}=Z^{i+}=\frac{\tilde{D}_{i}-\exp \left(-\beta_{i} e_{i}\right)}{\tilde{C}_{i}}+\frac{\tilde{A}_{i+1}-\exp \left(-\beta_{i+1} e_{i+1}\right)}{\tilde{C}_{i+1}} \\
& \left(\tilde{Z}_{3}\right)_{i}=\left(Z_{3}\right)_{i} \exp \left(\beta_{i} e_{i}\right)
\end{aligned}
$$

Using boundary conditions at both sides of the composite layer, relation (25) leads to a linear system whose unknowns are the $\theta^{i}$ $(i=1, \ldots,+\mathrm{N})$ :

$$
(\mathbf{C}+\mathbf{M}) \boldsymbol{\Theta}=\boldsymbol{\Psi}
$$




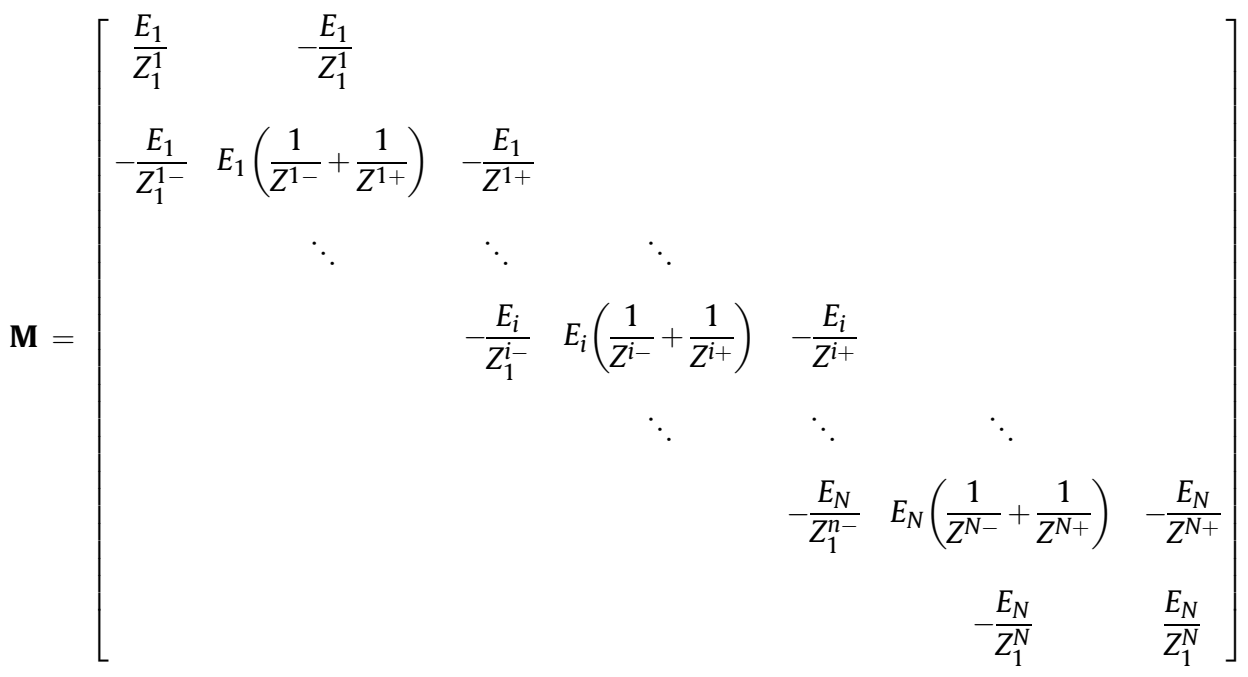

With: $E_{i}=\exp \left(-\beta_{i} e_{i}\right)$ and:

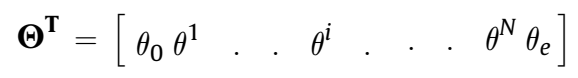

$\mathbf{C}=\left[\begin{array}{lllllll}h_{F} & & & & & & \\ & \frac{1}{\left(\tilde{Z}_{3}\right)_{1}} & & & & & \\ & & & \frac{1}{\left(\tilde{Z}_{3}\right)_{i}} & & & \\ & & & \ddots & \frac{1}{\left(\tilde{Z}_{3}\right)_{n}} & \\ & & & & & h_{R}\end{array}\right]$

$\Psi=\left[\begin{array}{c}\Psi_{0}(p) E_{1} \\ \vdots \\ Y_{i}(p) E_{i} \\ \vdots \\ \vdots \\ \Psi_{e}(p) E_{N}\end{array}\right]$

This formulation allows solving easily any multilayered problem whatever the heat source location. Nevertheless, even if the formulation of the mathematical problem is a tridiagonal linear system, such system is exactly solved by a Gauss method. Then, this solution is strictly explicit as classical analytic formulation. The main drawback comes when the number of layer increases so that the expression becomes very long and difficult to write.

\section{Application on two multilayered materials}

The application of this formalism is proposed with three examples of multilayered materials whose sketches are defined in Figs. 5-7 respectively. The representation with impedances is also used to illustrate the proposed decomposition. Thermophysical properties and geometric characteristics are given in Table 1.
5.1. Example 1: two layers with internal source and adiabatic conditions

Let us consider a multilayered wall with two layers of different thickness and materials. An internal heating characterized by a volume heat source $g$ that can vary only in time is located on the first layer and no flux are considered on the rear and front faces (insulated wall). The temperatures at the front face, the rear face and at the interface between the two materials are calculated considering the heat source as a Dirac function. In that case, the analytical expression for each is:

$$
\begin{gathered}
\theta_{0}=\frac{Y \exp \left(-\beta_{1} e_{1}\right)\left(\exp \left(-\beta_{2} e_{2}\right)+\tilde{Z}^{1+} \tilde{C}_{2}\right)}{\tilde{C}_{1}\left(\exp \left(-\beta_{2} e_{2}\right)+\tilde{Z}^{1+} \tilde{C}_{2}\right)+\tilde{C}_{2} \exp \left(-\beta_{1} e_{1}\right)} \\
\theta_{1}=\frac{Y \exp \left(-\beta_{1} e_{1}\right)\left(\exp \left(-\beta_{2} e_{2}\right)+\left(\tilde{Z}_{2}\right)_{1} \tilde{C}_{2}\right)}{\tilde{C}_{1}\left(\exp \left(-\beta_{2} e_{2}\right)+\tilde{Z}^{1+} \tilde{C}_{2}\right)+\tilde{C}_{2} \exp \left(-\beta_{1} e_{1}\right)} \\
\theta_{e}=\frac{Y \exp \left(-\beta_{1} e_{1}\right) \exp \left(-\beta_{2} e_{2}\right)}{\tilde{C}_{1}\left(\exp \left(-\beta_{2} e_{2}\right)+\tilde{Z}^{1+} \tilde{C}_{2}\right)+\tilde{C}_{2} \exp \left(-\beta_{1} e_{1}\right)}
\end{gathered}
$$

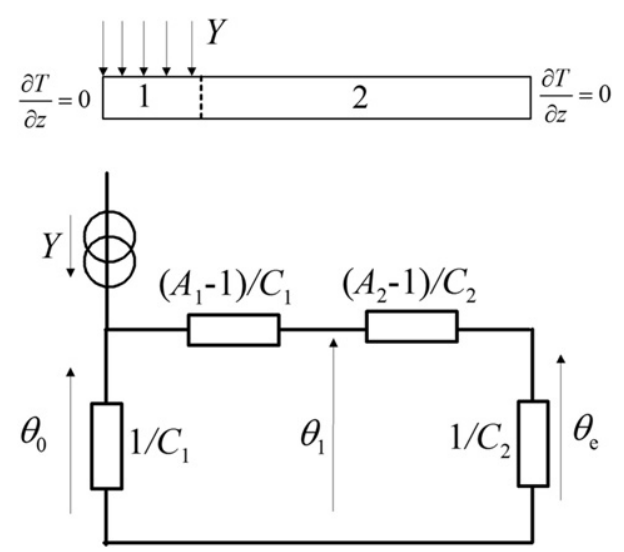

Fig. 5. Example 1 - Multilayered material (two layers) and zero exit fluxes (insulated wall). 

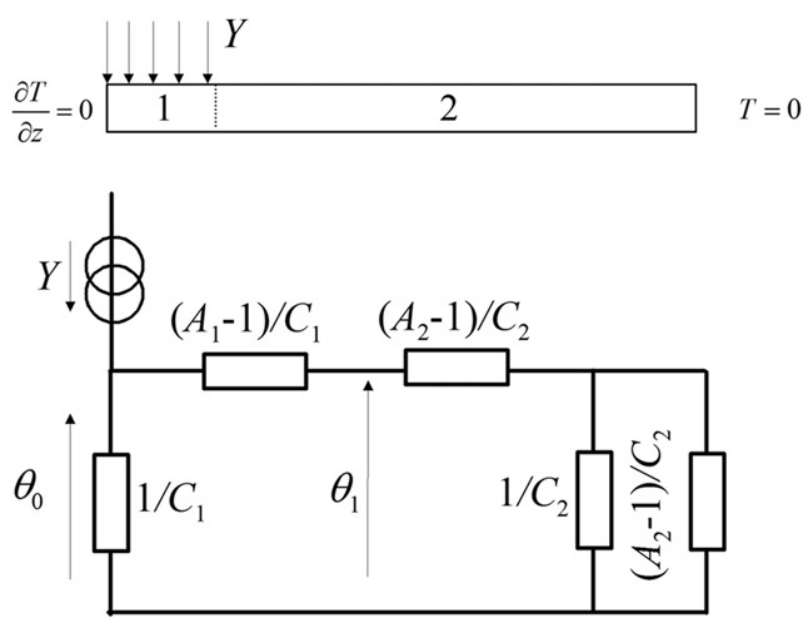

Fig. 6. Example 2-Multilayered material (two layers) and fixed temperature $\left(\theta_{e}=0\right)$.
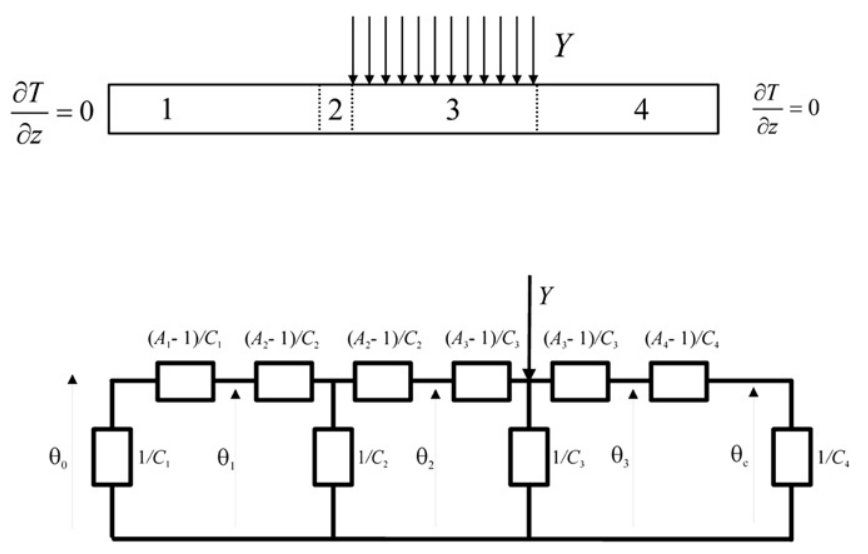

Fig. 7. Example 3 Multilayered materials (four layers) with internal source.

It is used the Den Iseger algorithm for the Laplace numerical inversion [21]. CPU times do not exceed $40 \mathrm{~s}$. Solution is represented in Fig. 8 in a log-log scale. It is obtained a satisfying result with regards to the asymptotic behaviours considering six decades for the time. Indeed, at the small times, the heat source term is equivalent to an initial temperature field in the layer 1 that is:

$T_{1 \text { init }}=\frac{Y}{\rho_{1} c_{1} e_{1}}$

Moreover, at the small times, the temperature $T_{1}$ at the interface is the contact temperature of two walls with two different initial temperatures:

$T_{1}=\frac{\sqrt{\lambda_{1} \rho_{1} c_{1}} T_{1 \text { init }}+\sqrt{\lambda_{2} \rho_{2} c_{2}} T_{2 \text { init }}}{\sqrt{\lambda_{1} \rho_{1} c_{1}}+\sqrt{\lambda_{2} \rho_{2} c_{2}}}=\frac{\sqrt{\lambda_{2} \rho_{2} c_{2}} \frac{Y}{\lambda_{1} \rho_{1} c_{1}}}{\sqrt{\lambda_{1} \rho_{1} c_{1}}+\sqrt{\lambda_{2} \rho_{2} c_{2}}}$

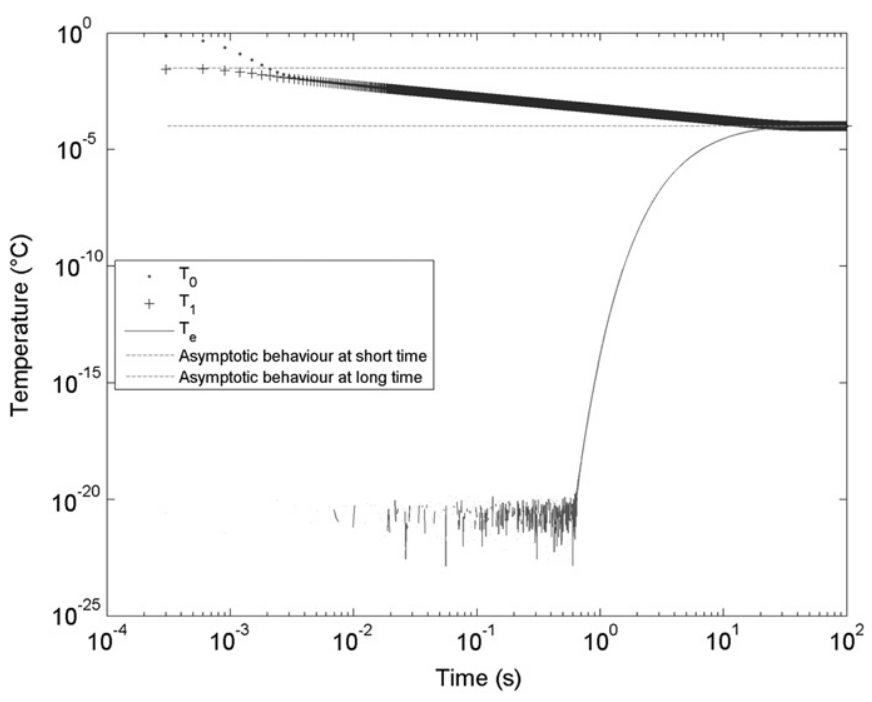

Fig. 8. Time representation of temperatures for example 1 .

The comparison between this value and the simulation shows a good agreement for small times range.

At the long times, all temperatures in the multilayered materials tend towards the same constant temperature:

$T_{1} \approx T_{0} \approx T_{e} \approx \frac{Y}{\lambda_{1} \rho_{1} c_{1}+\lambda_{2} \rho_{2} c_{2}}$

It is also well retrieved the $-1 / 2$ slope (in $\log -\log$ ) for the temperature at the front face and at the interface between the 2 layers for intermediate times. It is consistent with the semi-infinite behaviour.

\subsection{Example 2: two layers with internal source and imposed temperature}

Let us consider an identical multilayered material as in example 1, but with different boundary conditions. Temperature $T_{e}$ is maintained to a constant value equal to $0{ }^{\circ} \mathrm{C}$. Equivalent thermal impedances network presented in Fig. 6 allow obtaining analytical expressions of temperature at every location. The result is presented in Fig. 9 for temperatures $T_{0}$ and $T_{1}$ (at the front and rear faces respectively) along with their asymptotic behaviours. It is obviously obtained the same behaviour at the small times than that presented in previous example. When time increases, temperatures decrease to reach the prescribed temperature in stationary configuration. It is verified that the slope is still equal to $-1 / 2$. As previously, the numerical computation remains efficiently whatever the time range.

\subsection{Example 3: multilayer with internal source}

Let us consider a multilayered material constituted from four layers of different thickness and thermophysical properties. A source term on a form of a Dirac function is located in the third

Table 1

Definition of the three examples.

\begin{tabular}{|c|c|c|c|c|c|c|c|c|c|c|c|c|c|}
\hline \multirow[t]{2}{*}{ Layer } & \multirow[t]{2}{*}{$Y\left(\mathrm{~W} \mathrm{~m}^{-2}\right)$} & \multicolumn{3}{|l|}{1} & \multicolumn{3}{|l|}{2} & \multicolumn{3}{|l|}{3} & \multicolumn{3}{|l|}{4} \\
\hline & & $e(\mathrm{~m})$ & $\rho c\left(\mathrm{~J} \mathrm{~m}^{-3} \mathrm{~K}^{-1}\right)$ & $\lambda\left(\mathrm{W} \mathrm{m} \mathrm{m}^{-1} \mathrm{~K}^{-1}\right)$ & $e$ & $\rho c$ & $\lambda$ & $e$ & $\rho c$ & $\lambda$ & $e$ & $\rho c$ & $\lambda$ \\
\hline Example1 & 10 & $10^{-5}$ & $10^{6}$ & 0.1 & $10^{-1}$ & $10^{6}$ & 100 & & & & & & \\
\hline Example2 & 10 & $10^{-5}$ & $10^{6}$ & 0.1 & $10^{-1}$ & $10^{6}$ & 100 & & & & & & \\
\hline Example3 & $10^{6}$ & $10^{-2}$ & $10^{6}$ & 0.1 & $10^{-3}$ & $10^{6}$ & 1 & $10^{-9}$ & $10^{6}$ & 100 & $10^{-2}$ & $10^{6}$ & 0.1 \\
\hline
\end{tabular}




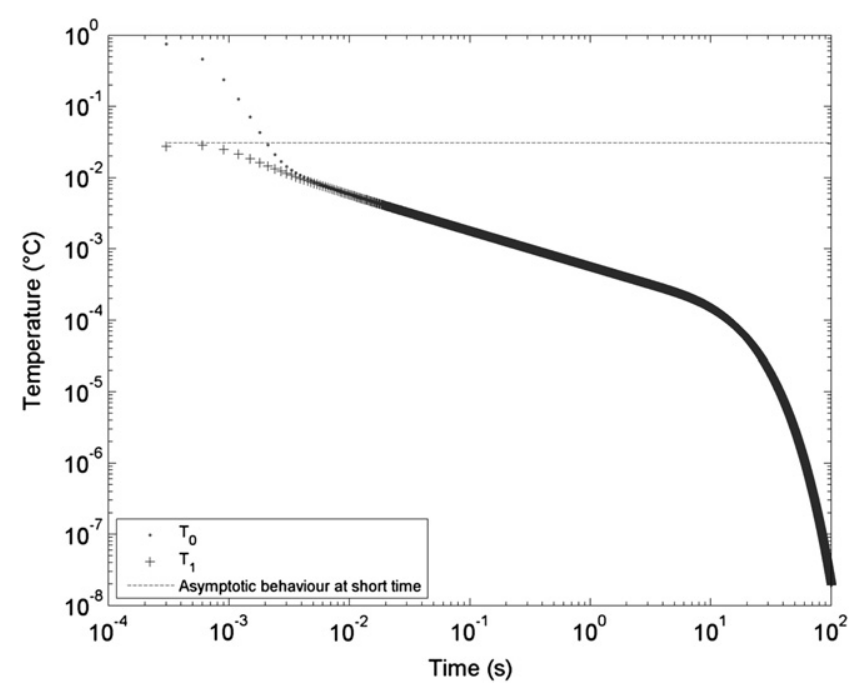

Fig. 9. Time representation of temperatures for example 2.

layer. In this case and with this new method, the analytical solution of temperatures in Laplace domain is easy to calculate but the explicit expression is quite difficult to write due to the number of layers. Nevertheless, as represented in Fig. 10, the interfacial temperatures can be efficiently calculated at the small times as well as the long times (Fig. 10).

A very interesting use can be the determination of thermophysical properties coupling this formalism with experimental devices and inverse methodologies.

\section{Conclusion}

An improvement of the thermal quadrupoles method for multilayered with and without heat sources has been presented in this paper. Numerical problems occur when using the classical thermal quadrupoles based on the use of hyperbolic functions. These problems depend on thermophysical and geometrical properties and observation times. It is proposed a new mathematical formulation based on the exclusive use of the exponential function with negative argument. As a consequence, the thermal impedances representation is also updated and lead to a very

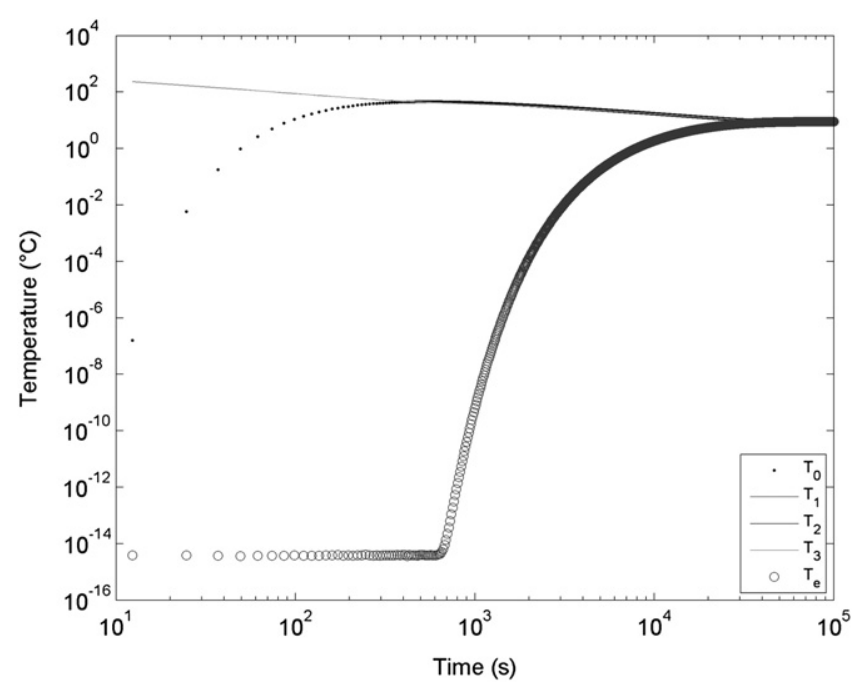

Fig. 10. Time representation of temperatures for example 3. efficient tool to model heat transfer in mulilayered with heat sources located in one or several layers. These applications demonstrate the ability of the technique to deal with broad ranges of time and thicknesses of the layers. Our methodology presents a real advantage considering what could have happened with a classical finite elements simulation because we can model problems with different scales. This accurate formulation has been already applied in thermal characterization problems even if it is tricky to have an explicit analytical solution when the number of layers becomes very important.

\section{References}

[1] D. Maillet, S. André, J.-C. Batsale, A. Degiovanni, C. Moyne, Thermal Quadrupoles: Solving the Heat Equation through Integral Transforms. John Wiley and Sons, New-York, 2000.

[2] A. Kusiak, C. Pradere, J.L. Battaglia, Measuring the thermal conductivity of liquids using photo-thermal radiometry, Measurement Science Technology. ISSN: 0957-0233 21 (1) (2009). ISSN: 0957-0233 6.

[3] A. Salazar, R. Celorio, Propagation of thermal waves in multilayered cylinders using thermal quadrupole method, The European Physical Journal, Special Topics 153 (2008) 383-386.

[4] R. Celorio, A. Mendioroz, E. Apinaniz, A. Salazar, C. Wang, A. Mandelis, Reconstruction of radial thermal conductivity depth in case hardened steel rods, Journal of Applied Physics 105 (8) (2009) 083517.

[5] N. Madariaga, A. Salazar, Propagation of thermal waves in multilayered spheres, Journal of Applied Physics 101 (103534) (2007) 1-7.

[6] M. Lazard, P. Corvisier, Modelling of tool during turning analytical prediction of the temperature and of the heat flux at the tool's tip. Applied Thermal Engineering 24 (2004) 839-849.

[7] S. Andre, B. Rémy, D. Maillet, A. Degiovanni, Modulated photothermal radiometry applied semitransparent samples: models and experiments, Journal of Applied Physics 96 (5) (2004) 2566-2575.

[8] Y. Jannot, Z. Acem, A. Kanmogne, Transient hot plate method with two temperature measurements for thermal characterization of metals, Measurement Science and Technology 17 (2006) 69-74.

[9] Y. Jannot, P. Meukam, Simplified estimation method for the determination of the thermal effusivity and thermal conductivity using a low cost hot strip, Measurement Science and Technology 15 (2004) 1932-1938.

[10] F. Volle, S.V. Garimella, M.A. Juds, A Thermal Quadrupole-based Model for Heat Diffusion in a Multilayered System: Application to Determination of Transient Performance of a Medium-Voltage Soft Starter. ASME International Mechanical Engineering Congress and Expoistion, IMECE, Boston, 2008, 31 October 2008 through 6 November, 2008.

[11] O. Fudym, C. Pradere, J.-C. Batsale, An analytical two-temperature model for convection-diffusion in multilayered systems: application to the thermal characterization of microchannel reactors, Chemical Engineering Science 62 (2007) 4054-4064.

[12] G.E. Cossali, Periodic heat conduction in a solid homogeneous finite cylinder International Journal of Thermal Science 48 (2009) 722-732.

[13] T. Kabayabaya, F. Yu, X. Zhang, Thermal diffusivity of glass at high temperature by using flash method, Journal of Thermal Science 13 (1) (2004).

[14] G. Wei, X. Zhang, F. Yu, K. Chen, Thermal diffusivity measurements on insulation materials with the laser flash method, International Journal of Thermophysics 27 (1) (2006) 235-243.

[15] V. Boudenne, L. Ibos, E. Gehin, Y. Candau, A simultaneous characterization of thermal conductivity and diffusivity of polymer materials by a periodic method, Journal of Physics D: Applied Physics 37 (2004) $132-139$.

[16] F. Albouchi, F. Mzali, S. Ben Nasrallah, Measurement of the effective thermal conductivity of powders using a three-layer structure, Journal of Porous Media 10 (6) (2007) 537-550.

[17] Z. Cheheb, F. Albouchi, S. Ben Nasrallah, Measurement of thermal radiative and conductive properties of semitransparent materials using a photothermal crenel method, Journal of Quantitative Spectroscopy \& Radiative Transfer 109 (2008) 620-635.

[18] A. Adili, C. Kerkeni, S. Ben Nasralla, Estimation of thermophysical properties of fouling using inverse problem and its impact on heat transfer efficiency, Solar Energy 83 (2009) 1619-1628.

[19] A. Degiovanni, Conduction dans un mur multicouche avec sources: extension de la notion de quadripôle, International Journal of Heat and Mass Transfer 31 (1988) 553-557.

[20] J.-C. Batsale, D. Maillet, A. Degiovanni, Extension de la méthode des quadripôles thermiques à l'aide de transformations intégrales - calcul du transfert thermique au travers d'un défaut plan bidimensionnel, International Journal of Heat and Mass Transfer 37 (1) (1994) 111-127.

[21] J. Toutain, J.-L. Battaglia, C. Pradere, J. Pailhes, A. Kusiak, W. Aregba, J.C. Batsale, numerical inversion of Laplace transform for time resolved thermal characterization experiment, Journal of Heat Transfer 133 (4) (2011) 044504 